\title{
Genome-wide characterization of a SRO gene family involved in response to biotic and abiotic stresses in banana (Musa spp.)
}

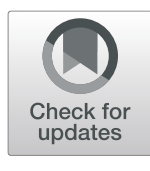

Lu Zhang ${ }^{1,2}$, Dengbo Zhou ${ }^{2}$, Huigang $\mathrm{Hu}^{3}$, Weiming $\mathrm{Li}^{3}$, Yulin $\mathrm{Hu}^{3}$, Jianghui Xie ${ }^{2,3}$, Shangzhi Huang ${ }^{1,4^{*}}$ and Wei Wang ${ }^{2 *}$

\begin{abstract}
Background: Banana (Musa spp.) is one of the world's most important fruits and its production is largely limited by diverse stress conditions. SROs (SIMILAR TO RCD-ONE) have important functions in abiotic stress resistance and development of plants. They contain a catalytic core of the poly(ADP-ribose) polymerase (PARP) domain and a Cterminal RST (RCD-SRO-TAF4) domain. In addition, partial SROs also include an N-terminal WWE domain. Although a few of SROs have been characterized in some model plants, little is known about their functions in banana, especially in response to biotic stress.

Results: Six MaSRO genes in banana genome were identified using the PARP and RST models as a query. Phylogenetic analysis showed that 77 SROs from 15 species were divided into two structurally distinct groups. The SROs in the group I possessed three central regions of the WWE, PARP and RST domains. The WWE domain was lacking in the group II SROs. In the selected monocots, only MaSROs of banana were present in the group II. Most of MaSROs expressed in more than one banana tissue. The stress- and hormone-related cis-regulatory elements (CREs) in the promoter regions of MaSROs supported differential transcripts of MaSROs in banana roots treated with abiotic and biotic stresses. Moreover, expression profiles of MaSROs in the group I were clearly distinct with those observed in the group II after hormone treatment. Notably, the expression of MaSRO4 was significantly upregulated by the multiple stresses and hormones. The MaSRO4 protein could directly interact with MaNAC6 and MaMYB4, and the PARP domain was required for the protein-protein interaction.

Conclusions: Six MaSROs in banana genome were divided into two main groups based on the characteristics of conserved domains. Comprehensive expression analysis indicated that MaSROs had positive responses to biotic and abiotic stresses via a complex interaction network with hormones. MaSRO4 could interact directly with MaNAC6 and MaMYB4 through the PARP domain to regulate downstream signaling pathway.
\end{abstract}

Keywords: SRO family, Banana, Induced expression, Abiotic stress, Biotic stress, hormone treatment, Protein interaction

\section{Background}

Plants are persistently challenged by numerous biotic and abiotic environmental stresses. Multiple stress factors result in an extensive loss of agricultural production worldwide [1]. During evolution, plants have developed

\footnotetext{
* Correspondence: huangsz@mail.sysu.edu.cn; wangweisys@ahau.edu.cn ${ }^{1}$ State Key Laboratory of Biocontrol and Guangdong Key Laboratory of Plant Resources, School of Life Sciences, Sun Yat-sen University, Guangzhou 510006, China

${ }^{2}$ Institute of Tropical Bioscience and Biotechnology, Chinese Academy of Tropical Agricultural Sciences, Haikou 571101, China

Full list of author information is available at the end of the article
}

sophisticated mechanisms to protect themselves against multiple stresses. Several molecules, such as transcription factors (TFs), reactive oxygen species (ROS), cytosolic $\mathrm{Ca}^{2+}$ and kinases, are involved in different stress signaling pathways $[2,3]$. Additionally, hormone signaling pathways regulated by salicylic acid (SA), jasmonic acid (JA), abscisic acid (ABA) and ethylene also play key roles in crosstalk between biotic and abiotic stresses [4-6].

A SIMILAR TO RCD ONE (SRO) family is a group of plant-specific proteins, which participate in abiotic stress

(C) The Author(s). 2019 Open Access This article is distributed under the terms of the Creative Commons Attribution 4.0 International License (http://creativecommons.org/licenses/by/4.0/), which permits unrestricted use, distribution, and 
and developmental processes [7]. In Arabidopsis thaliana, a radical-induced cell death 1 (AtRCD1) was originally discovered according to the recovery of oxidative stress response defects in yeast mutant [8]. Subsequently, homologs (AtSRO1-5) of AtRCD1 were also identified and divided into two structural types [7]. AtRCD1 and AtSRO1 in the type I contain an N-terminal WWE domain (PS50918), a poly(ADP-ribose) polymerase (PARP) domain (PS51059), and a C-terminal RCD1-SRO-TAF4 (RST) domain (PF12174) [9]. The type II includes AtSRO2, 3, 4, and 5 lacking the WWE domain [7]. Previous studies demonstrated that the RST domain was specifically involved in plant SRO and TAF4 proteins, while the WWE-PARP domain widely existed in various organisms $[10,11]$. The WWE domain might be required for protein-protein interactions by forming a globular structure [12]. The RST domain is essential for the SRO interaction with different TFs [7, 9].

Until now, limited knowledge of SROs was obtained from Arabidopsis and rice. A loss-of-function mutation of AtRCD1 resulted in the increased sensitivity to the abiotic stress responses, the aberrant leaf and rosette morphology, and the altered hormone responses [1316]. AtRCD1 was also involved in salt stress by interacting with SOS1, a plasma membrane $\mathrm{Na}^{+} / \mathrm{H}^{+}$antiporter [17]. However, little work had been done on AtSRO2, 3, 4 and 5. AtSRO2 was upregulated in response to light treatment. AtSRO3 was significantly downregulated under light stress, but induced by salt stress and ozone [7]. The mutated AtSRO5 plants were more sensitive to $\mathrm{H}_{2} \mathrm{O}_{2}$-mediated oxidative stress and salt stress [18]. In addition, a rice SRO protein OsSRO1c had dual roles in improving drought and oxidative stress tolerance by the interaction with NAC1 and zinc finger TFs $[19,20]$. The wheat SRO could alleviate the oxidative stress under salt treatment by modulating redox homeostasis [212]. Likewise, overexpression of apple $R C D 1$ in transgenic apple calli and Arabidopsis plants enhanced the plant resistance to salt stress [22]. However, whether members of the $S R O$ gene family also participate in the regulation of biotic stress is still an open question.

Although some functional studies on this family have been carried out in the model plants, very little is known in the non-model plants. Banana is vital for food security in many tropical and subtropical countries. The vegetative propagation of banana commercial cultivars resulted in the susceptibility to pests and diseases due to the narrow genetic background [23]. For example, fusarium wilt, caused by Fusarium oxysporum f. sp. cubense (Foc), is one of the most destructive diseases, which can cause leaf wilt and death of the whole plant. Especially, a strain of Foc called tropical race 4 (Foc TR4) has overcome more than $80 \%$ of global banana and plantain [24]. Moreover, adverse environmental factors such as drought and low temperature also restrict the industrial development of global banana [25, 26]. To understand MaSRO function in response to abotic and biotic stresses, we first identified and characterized the SRO gene family of banana genome. Expression patterns of MaSROs were detected in response to six abiotic stresses (cold, osmotic, salinity, ultraviolet, heat and, wounding), one biotic stress (Foc TR4 inoculation), and four hormone treatments (ABA, SA, GA, and ethylene). By contrast, MaSRO4 was upregulated by multiple stress responses and interacted with MaNAC6 and MaMYB4 by the PARP domain.

\section{Results}

\section{Identification and classification of MaSROs}

After searching the entire genome of banana using the PARP and RST models, six MaSROs were obtained and named sequentially from MaSRO1 to MaSRO6 according to the description of Jaspers et al. (2010) [7]. A Maximum-Likelihood (ML) phylogenetic tree was produced using the full-length protein sequences of MaSROs. Six MaSROs clustered into two distinct groups (Fig. 1a). The group I contained four members from MaSRO1 to MaSRO4 with the WWE, PARP and RST domains, while MaSRO5 and MaSRO6 lacking the WWE domain belonged to the group II. To further identify other conserved domains, we analyzed all amino-acid (AA) sequences of MaSROs against the MEME tool [22]. In total, seven conserved motifs were listed in Additional file 1. MaSRO5 and MaSRO6 evidently lacked the internal motif I and motif VI, whereas motif VI was only found in MaSRO2 (Fig. 1b,c). Two highly conserved Motifs V and VII in the C-termini of MaSROs harbored the typical PARP and RST domains, respectively. Motif I within the WWWE domain mainly located in the $\mathrm{N}$-termini. The structure distribution of motifs supported the grouping results (Fig. 1a).

To determine the genomic structures of MaSROs, each DNA sequence was used to search the banana-genome database. Distributions of MaSROs on banana 12 chromosomes seemed to be uneven (Additional file 2). Chromosomes 4 and 5 contained two MaSROs, respectively. MaSRO2 and MaSRO4 located on chromosome 7 and 9, respectively. Exon/intron structures of MaSROs (such as between MaSRO3 and MaSRO4, between MaSRO1 and MaSRO2) were conserved within the same subfamily, except for MaSRO6 and MaSRO7 (Fig. 1d).

We also predicted the chemical and physical characters of each MaSRO protein. The lengths of MaSROs range from 240 to $490 \mathrm{AAs}$, and the values of GRAVY change from -0.202 to -0.435 . Based on an instability index, most of MaSROs belong to unstable proteins. MaSROs have obvious changes in isoelectric point from 


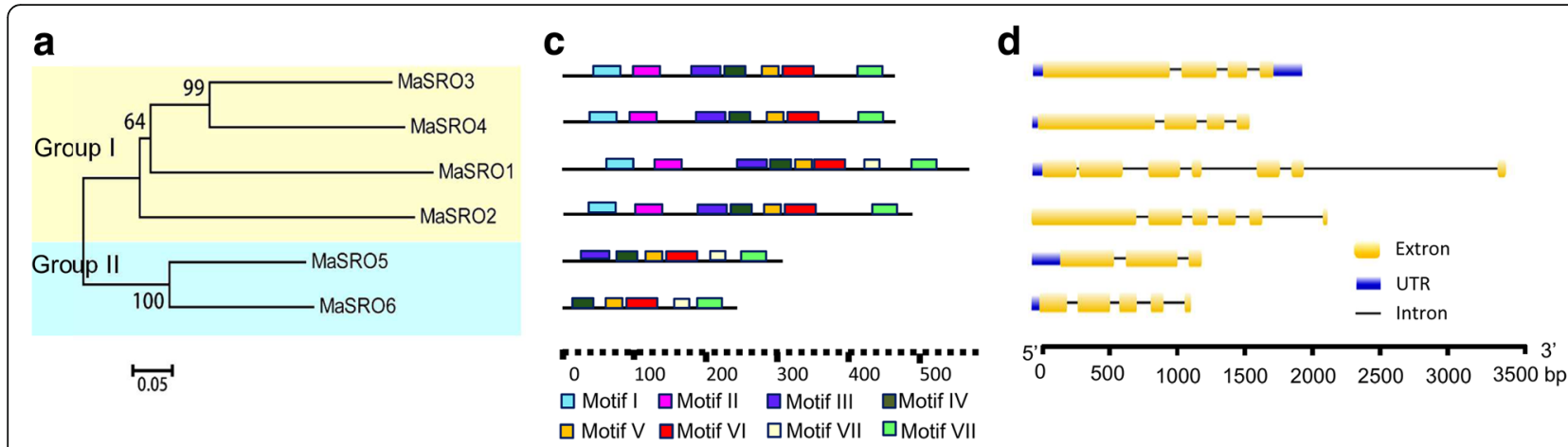

b

Motif I

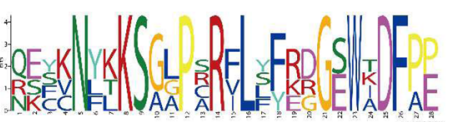

Motif IV

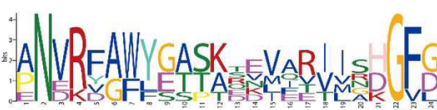

Motif VII

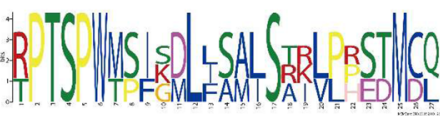

Motif II

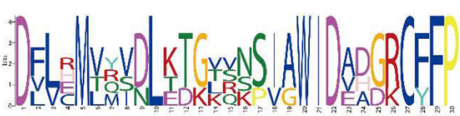

Motif V

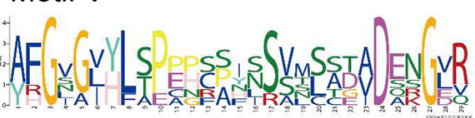

Motif VIII

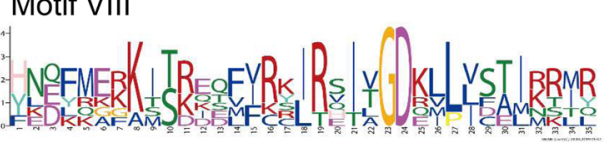

Fig. 1 Analysis of evolutionary relationship, protein domains and gene structures of the banana SRO gene family. a Six MaSROs clustered into two groups in an unrooted ML tree constructed by MEGA7.0. The group I contained MaSRO1, 2, 3 and 4. MaSRO5 and MaSRO6 formed the group II. b Seven conserved motifs of MaSROs were generated by the online MEME tool. The overall height of the stack represented the level of sequence conservation. Heights of residues within a stack indicated the frequency of each residue at the indicated position. c The positions of identified seven motifs were schematically presented in MaSROs. Different motifs were displayed using the different colored boxes. The lengths of proteins and motifs were estimated using the scale at the bottom. $\mathbf{d}$ The intron/exon structures of MaSROs were analyzed by comparing the genomic and cDNA sequences. Yellow and black boxes represented the exon and intron regions, respectively. Blue boxes indicated the untranslated regions (UTRs). The sizes of exons and introns were measured using the scale at the bottom

6.33 to 9.69 and in molecular weight from $27.023 \mathrm{kDa}$ to $66.147 \mathrm{kDa}$. The subcellular localizations of MaSRO1, 2, 3 and 5 were predicted in chloroplast or nucleus, whereas MaSRO4 and MaSRO 6 located in nucleus shown in Additional file 2.

\section{Phylogenetic analysis of the SRO gene family}

To investigate the evolutionary relationship among plant SRO proteins, we identified 80 SROs in 16 genome-sequenced species including A. thaliana (6), Zea mays (6), Populus trichocarpa (7), Musa acuminata (6), Solanum lycopersicum (6), Vitis vinifera (5), Physcomitrella patens (3), O. sativa (5), Medicago truncatula (7), Malus domestica (6), Brachypodium distachyon (5), Setaria italica (4), Chlamydomonas reinhardtii (2), Glycine max (5), Elaeis guineensis (3), and Phoenix dactylifera (3) in Additional file 3. The selected plants represented species within the division of angiospermae and Bryophyta (Fig. 2). The numbers of SROs in different plant species showed a gradual increase from algae to flowering plants along with the increase of organism complexity [27]. Most plants owned 5-7 SRO members in their genomes. E. guineensis, $P$. patens and $P$. dactylifera contained three SROs, while only two SRO homologs were identified in the $C$. reinhardtii genome. No SRO homologs were observed in a eukaryotic microalga Coccomyxa subellipsoidea and a unicellular green alga Ostreococcus lucimarinus. Likewise, we did not also found SRO homologs in the photosynthetic and/or eukaryotic microorganisms. Loss of ancestral SROs in specific lineages supports the evolutionary diversification through extensive expansion during plant evolution.

Based on the full-length protein sequences of SROs (Additional file 4), a ML phylogenetic tree was generated using bootstrap analysis (1000 replicates) (Additional file 5). Deviation seemingly happened from $C$. reinhardtii with a separate monophyletic clade, thereby confounding the tree topology. Hence, we removed two CrSROs from the final phylogenetic tree. A total of 77 SRO members from 15 species were clustered into the group I and II (Fig. 3). Based on the branch value ( $>75$ ) [28], the group I was further classified into four subgroups (Ia, Ib, Ic, and Id) [7] . AtRCD1 and AtSRO1 formed the subgroup Ia with the 


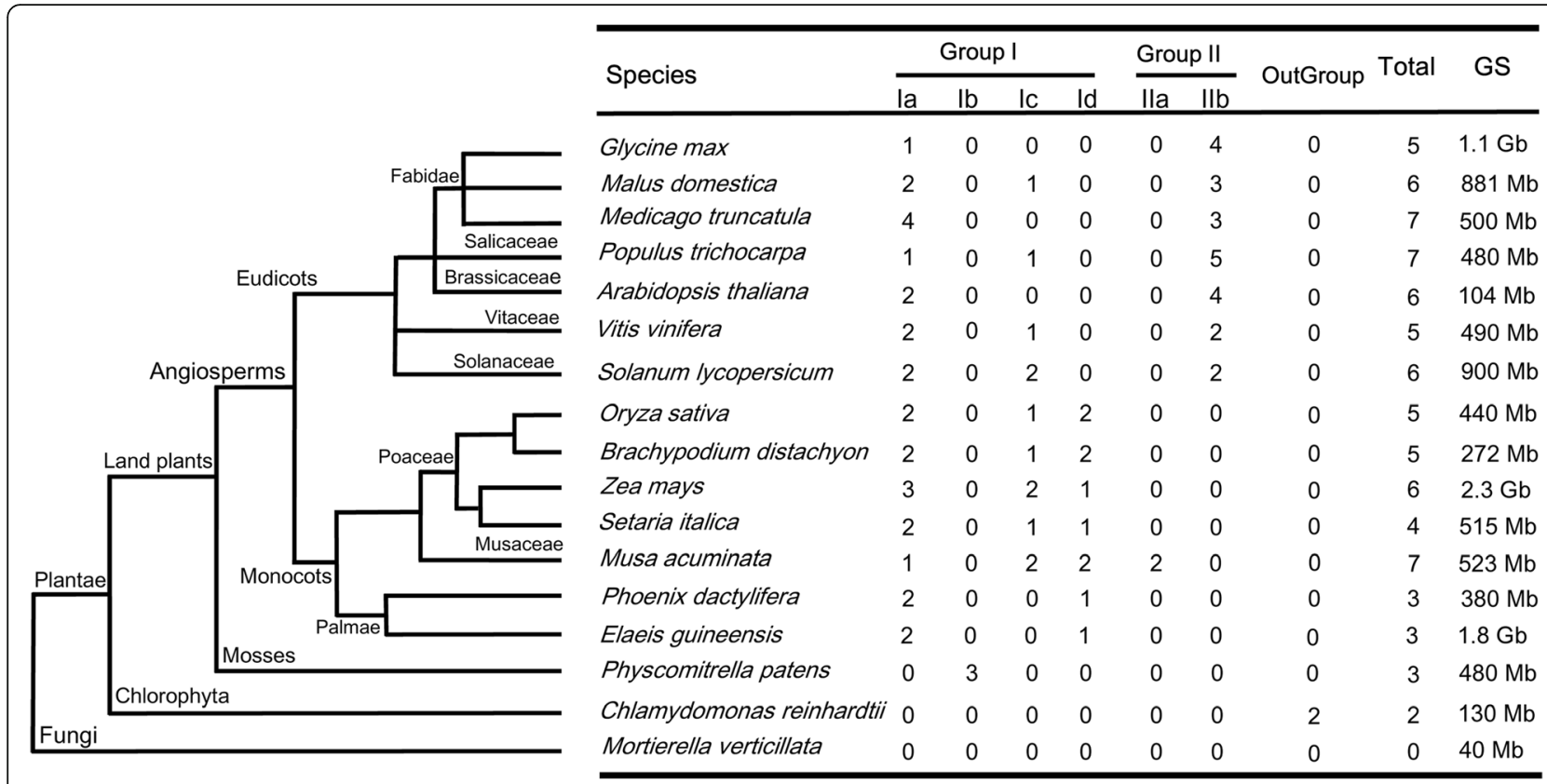

Fig. 2 Distribution of the SROs in Plantae. The left graph indicated the categories of species. The nodes of tree represented the evolutionary relationship. The number and classification of SROs in each species were described in the right diagram. GS: genome size

SRO homologs from all selected species except for $P$. patens, where an obvious divergence branch was produced between monocots and eudicots (Fig. 2). Three PpSROs of $P$. patens independently consisted of subgroup Ib. Only monocots in our selected species were involved in subgroup Id. The group II can be classified into one group. Besides MaSRO5 and MaSRO6 from banana, no SRO homologs of other monocots belonged to the subgroup II.

\section{Characteristics of conserved domains in plant SROs}

Based on the domain architecture, those SROs in the group I had a central region of the WWE, PARP and RST domains. No WWE domain was found in the SRO members of group II. Compared with compositions of AAs in three domains, an obvious variation was observed in the WWE domain (Additional file 6). The RST domain was most conserved in monocots. There are a strong conservation of aliphatic AAs in the $\mathrm{N}$-terminus, a strictly conserved tyrosine $(\mathrm{Y})$ in the middle of RST domain, and two glycine (G) and aspartic acid (D) residues in the C-terminus. In addition, seven conserved motifs were used to demonstrate the domain architecture of plant SROs from 15 species (Additional file 7). The basic PARP domain containing motif I and motif VII was found in all SROs, suggesting that this domain was important for basic function of SROs. It was supported that the phylogenetic trees constructed using the conserved PARP domain and full-length protein sequences were similar, although a difference was generated in the definition of subgroup
(Additional file 8). The motif II within the variable WWE region was shown in the group I, except for PpSRO1 from $P$. patens. Especially, most of members in the same subgroup shared one or more motifs outside the PARP and RST domains, further supporting the subgroup definition (Additional file 7).

Expression profiles of MaSROs in different banana tissues A quantitative real-time PCR (qRT-PCR) was performed to investigate expression patterns of MaSROs in banana (Musa AAA, cv. Williams) roots, leaves, stems, and fruits (Additional file 9). The PCR product of each MaSRO was confirmed by sequencing. Transcripts of MaSROs could be detected in the all selected four tissues, but low expression levels were observed in fruits. Remarkably, MaSRO2 exhibited relatively high expression levels in roots, while low transcript accumulation was detected in banana stems and leaves. MaSRO1, 3 and 4 clustering into the same group showed a preferential expression in stems. MaSRO5 had a constitutive expression in different organs. Our results suggest that MaSROs might play key roles in multiple biological processes during growth of banana plants.

\section{Expression characteristics of MaSROs under diverse abiotic stresses}

To identify potential functions of MaSROs in response to different abiotic stresses, their transcript profiles were assayed under polyethylene glycol (PEG), salt, cold, heat, ultraviolet (UV) and wounding treatments (Fig. 4). The 


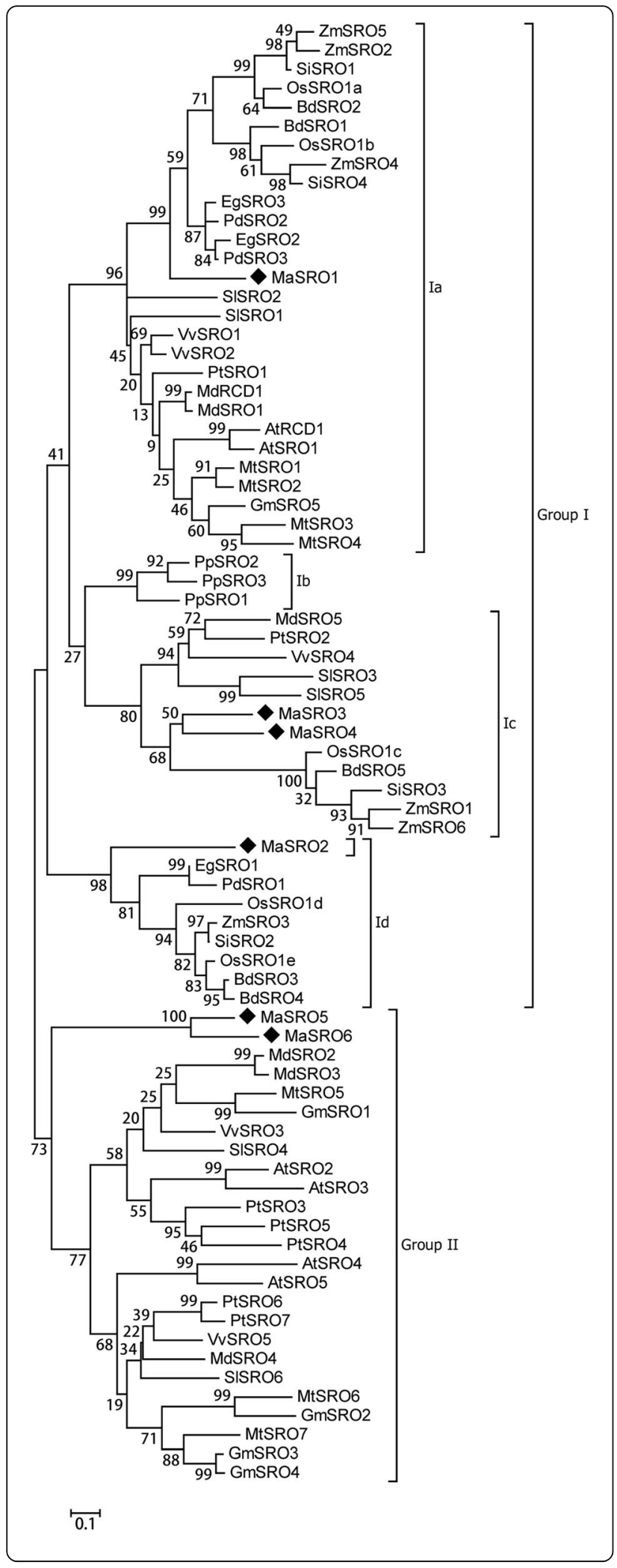

Fig. 3 Phylogenetic analysis of the SROs from different plants. A total of 77 SROs were identified from 15 representative plant species. The ML phylogenetic tree was constructed using the MEGA7 software based on the full-length protein sequences of SROs. Numbers on branches were bootstrap values calculated from 1000 replicates. These SROs were clustered into two main groups and five subgroups. The scale bar indicated AA substitutions per position

PEG treatment was used to simulate osmotic stress. At the initial stage, the expression levels of MaSROs were obviously upregulated except for MaSRO5. Especially, the transcript levels of MaSRO1, 3 and 4 gradually increased under PEG treatment until $24 \mathrm{~h}$. No significant difference was found in the transcription accumulation of MaSRO5 among the indicated time points. Under salt treatment, MaSRO3 and MaSRO4 reached an expression peak at $24 \mathrm{~h}$ and $12 \mathrm{~h}$, respectively, while other members were downregulated or showed no significant changes. By contrast, expression of MaSRO1, 3, 4 and 5 could be induced by heat treatment. The increase of MaSRO3 and MaSRO4 ranged from 5.41- to 11.76-folds and the enhancement of MaSRO1 and MaSRO5 were between 1.18- and 3.01-folds. Under cold stress, an obvious upregulation was observed in the expression levels of MaSRO1, 2, 3 and 4, but both MaSRO5 and MaSRO6 were down-regulated. Notably, the expression levels of MaSRO5 and MaSRO6 were induced at the early stage under UV stress, while the transcripts of other members were repressed. The wounding treatment induced rapidly the up-regulation of MaSRO3 and MaSRO4. Opposite results were detected in the expression patterns of MaSRO2. Hence, transcripts of these MaSROs were responsive to most of the applied stress treatments. Especially, MaSRO4 exhibited significant changes under multiple stress treatments, suggesting that it may own a unique role in stress responsiveness.

\section{Expression profiles of MaSROs in response to diverse hormone treatments}

Previous evidences indicated that different hormones play important roles in stress signal transduction and cell responses [4-6]. Here, we investigated the expression profiles of MaSROs in response to ABA, GA, ethylene, and SA treatments (Fig. 5). The highest numbers of MaSROs were induced by ABA treatment, followed by GA or ethylene treatment. Interestingly, SA treatment cannot significantly upregulated the expression levels of MaSROs, except for MaSRO3 and MaSRO4. MaSRO5 and MaSRO6 were more sensitive to ABA or GA treatment. Compared with abiotic stresses, MaSROs in the same subgroup showed analogous responses to exogenous hormones. For example, MaSRO2 from the subgroup Id displayed similar expression patterns after GA or ethylene treatment. We also found that the induced 

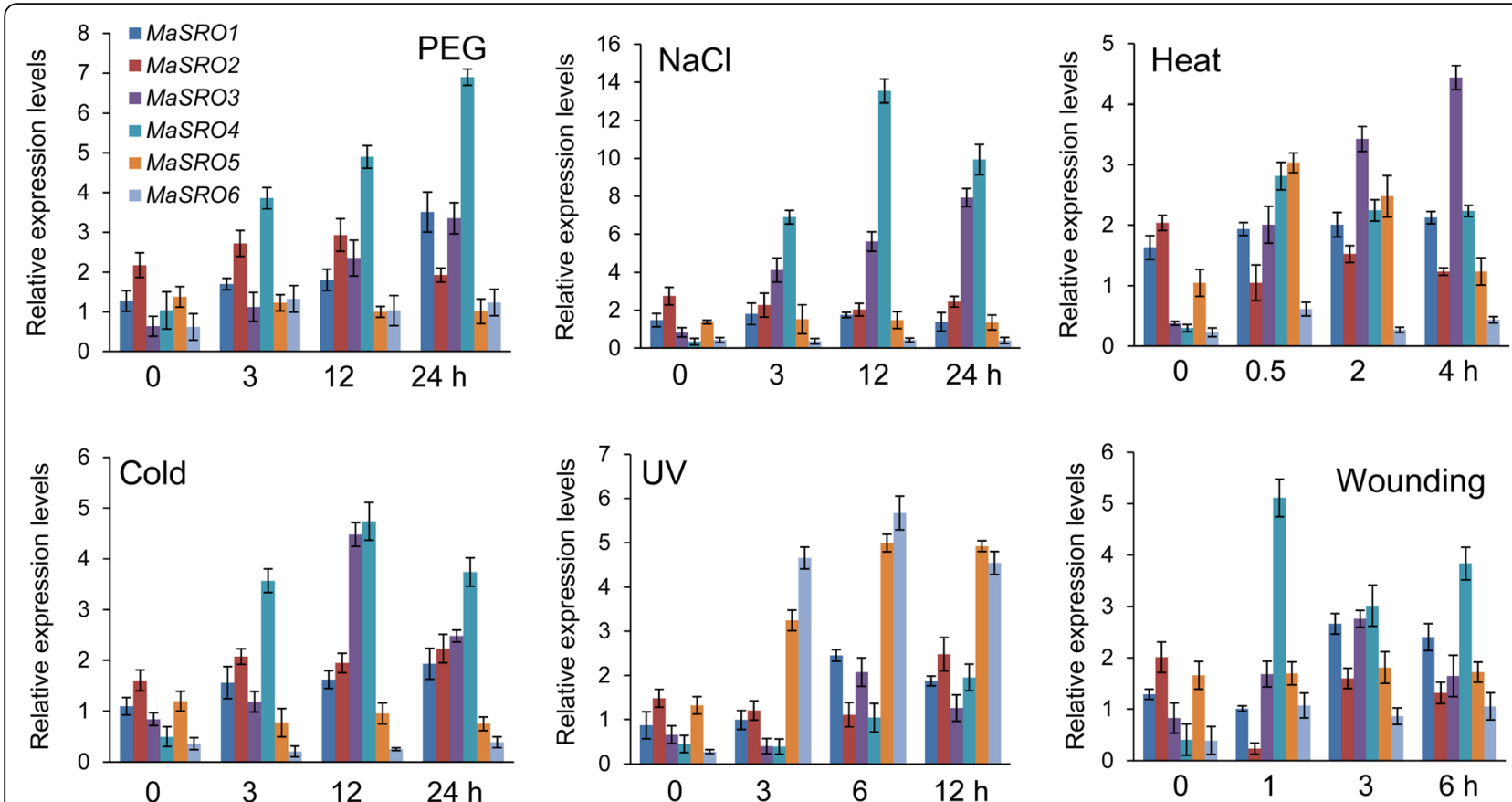

Fig. 4 qRT-PCR expression analysis for six MaSROs in banana roots under diverse abiotic stresses. The treated banana roots were harvested at the indicated time points. Data indicated relative expression levels (means \pm SE) from three independent biological replica (three RNA extractions; $n=3$ ). Used primers specific for MaSROs were listed in Additional file 10
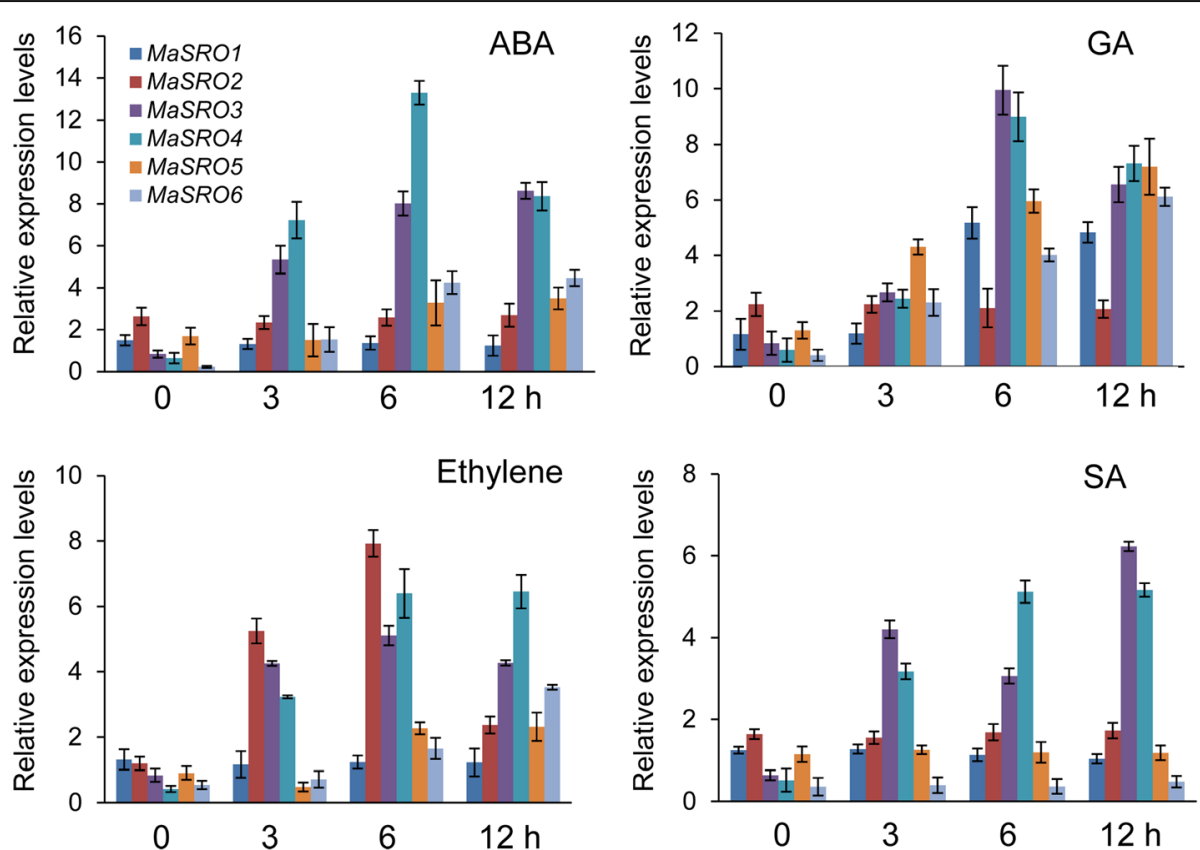

Fig. 5 Expression profiles of MaSROs under various hormone treatments by qRT-PCR. The treated banana roots were harvested at the indicated time points. Data indicate relative expression levels (means \pm SE) from three independent biological replica (three RNA extractions; $n=3$ ). Used primers specific for MaSROs were listed in Additional file 10 
transcript profiles of MaSROs in the group II were clearly different from those observed in the group I. MaSRO2, 3 and 4 in the group I showed an expression peak at the indicated time points after GA or ethylene treatment., MaSRO5 and MaSRO6 in the group II showed a continual transcript increase throughout the detected time points.

\section{Expression profiles of MaSROs in banana roots inoculated} with Foc TR4

To investigate whether MaSROs are required for biotic stress, their transcription levels were analyzed in banana roots after inoculation with Foc TR4 (Fig. 6). Except for MaSRO3 and MaSRO6, most of MaSROs increased by more than 2 -fold at $2 \mathrm{~h}$ post inoculation (hpi) in comparison with the initial stage. During Foc TR4 infection, the expression of MaSRO2 displayed a sharp increase, reached two peaks at $2 \mathrm{hpi}$ and $48 \mathrm{hpi}$ and then decreased in the following time points. MaSRO4 and MaSRO5 showed biphasic expression patterns with upregulation at $24 \mathrm{hpi}$ and downregulation at $48 \mathrm{hpi}$. The expression peaks of MaSRO4 and MaSRO5 were detected at $72 \mathrm{hpi}$. Both MaSRO3 and MaSRO6 demonstrated low or moderate levels in consecutive expression windows.

\section{Stress- and hormone-induced cis-regulatory element (CRE) analysis}

Transcriptional control of gene expression depends on CREs in the promoter region. In our study, the 1.5-kb upstream regions of the translational start sites of MaSROs were used for identification of CREs. Some stress- and hormone -related CREs were found according to their potential responsive functions (Fig. 7). More than one CRE were detected in the promoter region of each MaSRO. By contrast, most frequent CREs were identified, such as
MYCCONSENSUSAT (CANNTG, ABA response factor binding site), GT1GMSCAM4 (GAAAAA, ethylene response factor binding site), WBOXNTERF3 (TGACY, responsible for pathogen- and salt-induced expression), W-Box within ELRECOREPCRP1 (TTGAC, recognized specifically by SA-induced WRKY DNA-binding proteins), ASF1 MOTIFCAMV binding site (TGACG, involved in transcriptional activation by auxin and SA treatments), low-temperature responsive elements (CCGAAA, ACCG ACA and CCGAC), and auxin response factor binding sites (TGTCTC). There was an obvious difference in the type and abundance of CREs in six MaSROs promoters analyzed (Fig. 7). The HSE, W-box, and CBF/DREB1 elements were scattered in the promoter regions of all MaSROs. The promoter of MaSRO4 contained the most diverse collection of putative CREs (a total of 25 CREs, such as CBF/DREB1, LTR, HSE, GARE, SEBF, ABRE, W-box and ERF3, etc). Some hormone response elements including TACGTGTC (ABA), TAACGTA (GA), TCAT CTTCTT (SA), and AACGTG (JA) were specifically detected in the MaSRO4 promoter. In the MaSRO1 promoter, GA response elements such as TATCCAC, TAAC AAA and TATCCA were lacking when compared to MaSRO3 and MaSRO4. Only a small type of potential CREs were identified in the promoters of MaSRO1, MaSRO2 and MaSRO6. The GA and SA response elements were absent in the MaSRO6 promoter. By combining expression patterns of MaSROs with CER analysis, we found that MaSROs in response to multiple stimuli had a positive correlation with the types and numbers of CREs.

\section{Identification of interaction proteins with $\mathrm{MaSRO} 4$}

Earlier studies indicated that AtRCD1 and OsSRO1c could interact with numerous TFs (DREB2B, AP2/ERF, MYB, bZIP and NAC families) to activate stress

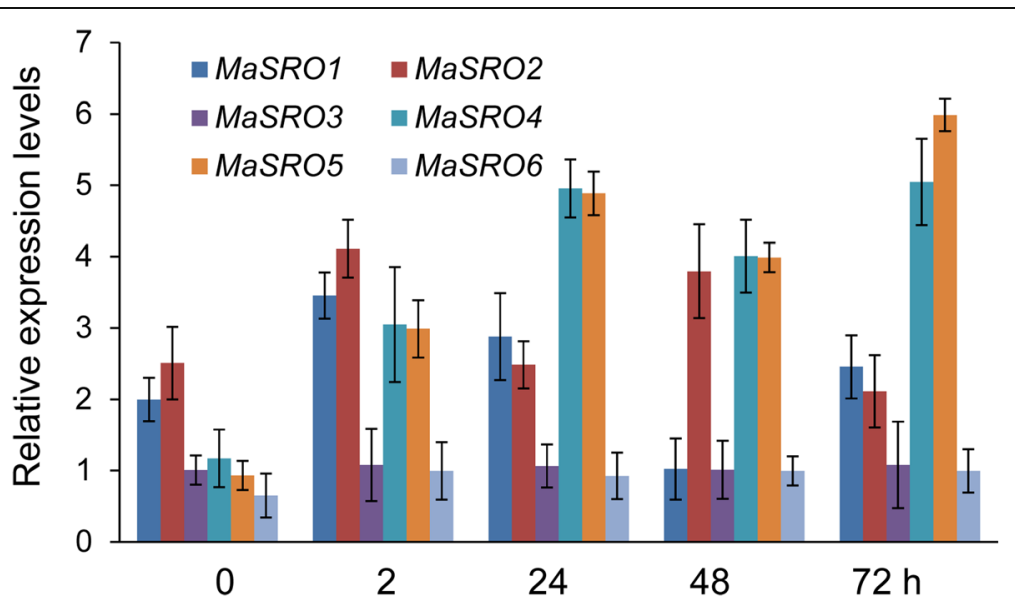

Fig. 6 qRT-PCR analysis of MaSROs in banana roots inoculated with Foc TR4. The samples were harvested at 0, 2, 24, 48 and $72 \mathrm{~h}$ after Foc TR4 inoculation. Data indicate relative expression levels (means \pm SE) from three independent biological replica (three RNA extractions; $n=3$ ). Used primers specific for MaSROs were listed in Additional file 10 


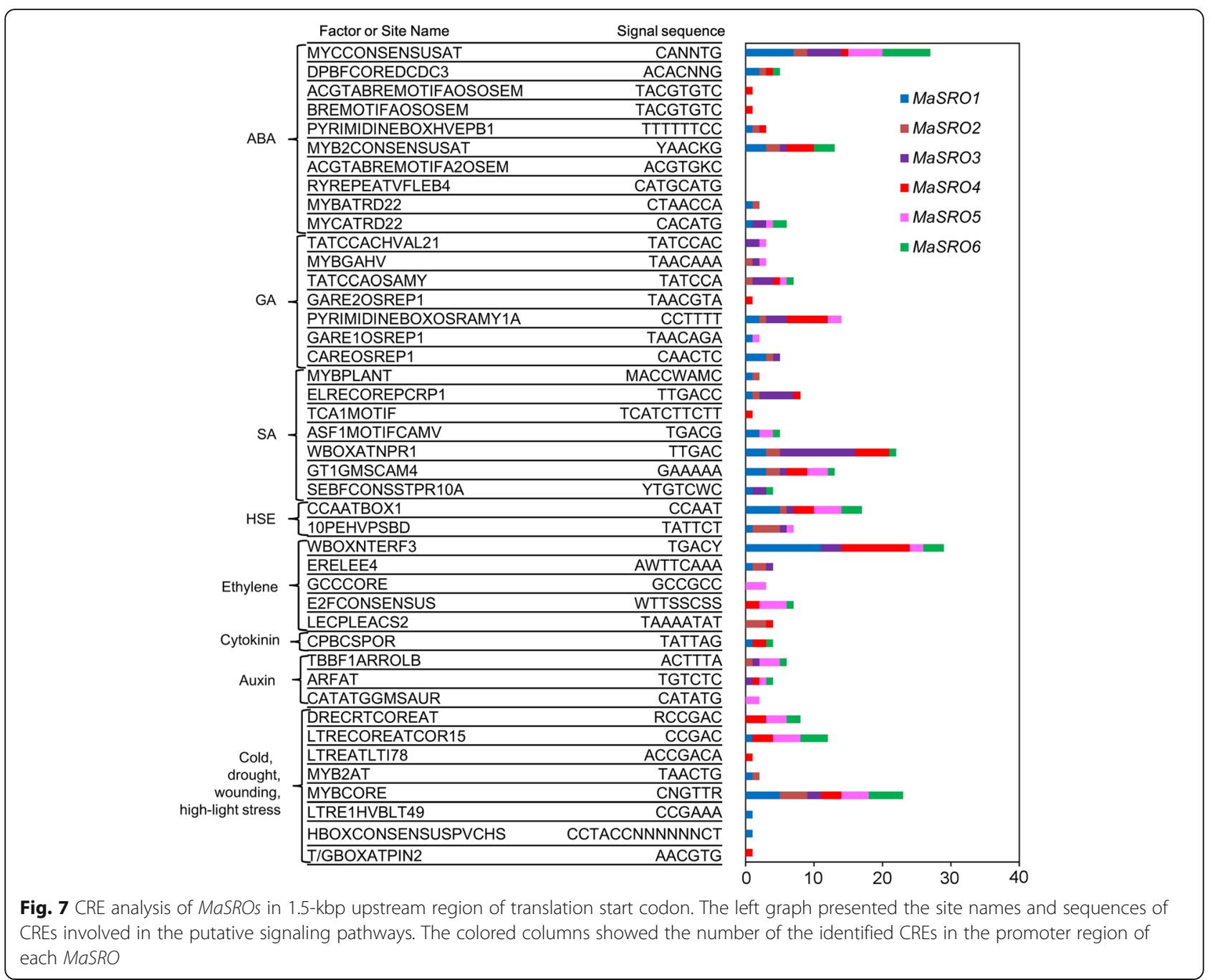

signaling pathways $[7,9,19,20]$. Because expression of MaSRO4 was significantly induced by multiple stress treatments, we further analyzed its interaction proteins to predict the stress-related regulation mechanism. Domain analysis uncovered the presence of a complete WWE-PARP-RST structure in MaSRO4, suggesting that the protein may also interact with homology TFs of rice or Arabidopsis. Here, yeast two-hybrid assay and immunoprecipitation (Co-IP) were performed to identify interaction proteins of MaSRO4. A MaSRO4-BD construct was used as a bait protein, and the selected banana TFs were constructed into the prey vector. By the growth of yeast on selection medium (lacking Leu, Trp, His, and Ade) and $\alpha$-galactosidase assay, the full-length MaSRO4 protein can directly interact with MaNAC6 and MaMYB4 (Fig. 8a). Furthermore, we co-expressed MaSRO4-3HA and TF-FLAG in Arabidopsis protoplasts. Anti-FLAG resin was used for Co-IPs (Fig. 8b). Western blot analysis with anti-3HA antibody showed that the precipitated fraction contained MaSRO4-3HA. Hence,
MaSRO4-3HA could be immuno-precipitated by MaNAC6-/MaMYB4-FLAG.

To understand whether the different domains of MaSRO4 are necessary for its interaction with MaMYB4, different $\mathrm{N}$ - and $\mathrm{C}$-terminal truncations were generated by decreasing the length of MaSRO4. MaSRO4 lacking the WWE domain did not substantially affect the interaction with MaMYB4 in yeast two-hybrid system (Fig. 8c), suggesting that the WWE domain was not necessary for protein interaction. However, it was not possible to test the RST domain alone in this system, because a C-terminal construct lacking both the WWE and PARP domains strongly auto-activated the expression of the reporter gene in yeast. Deletions of the PARP domain resulted in loss of interaction with MaMYB4, indicating that the domain was required for MaSRO4 function.

\section{Discussion}

Although SRO homologs widely exist in the kingdom of Plantae, only a few members from rice and Arabidopsis 


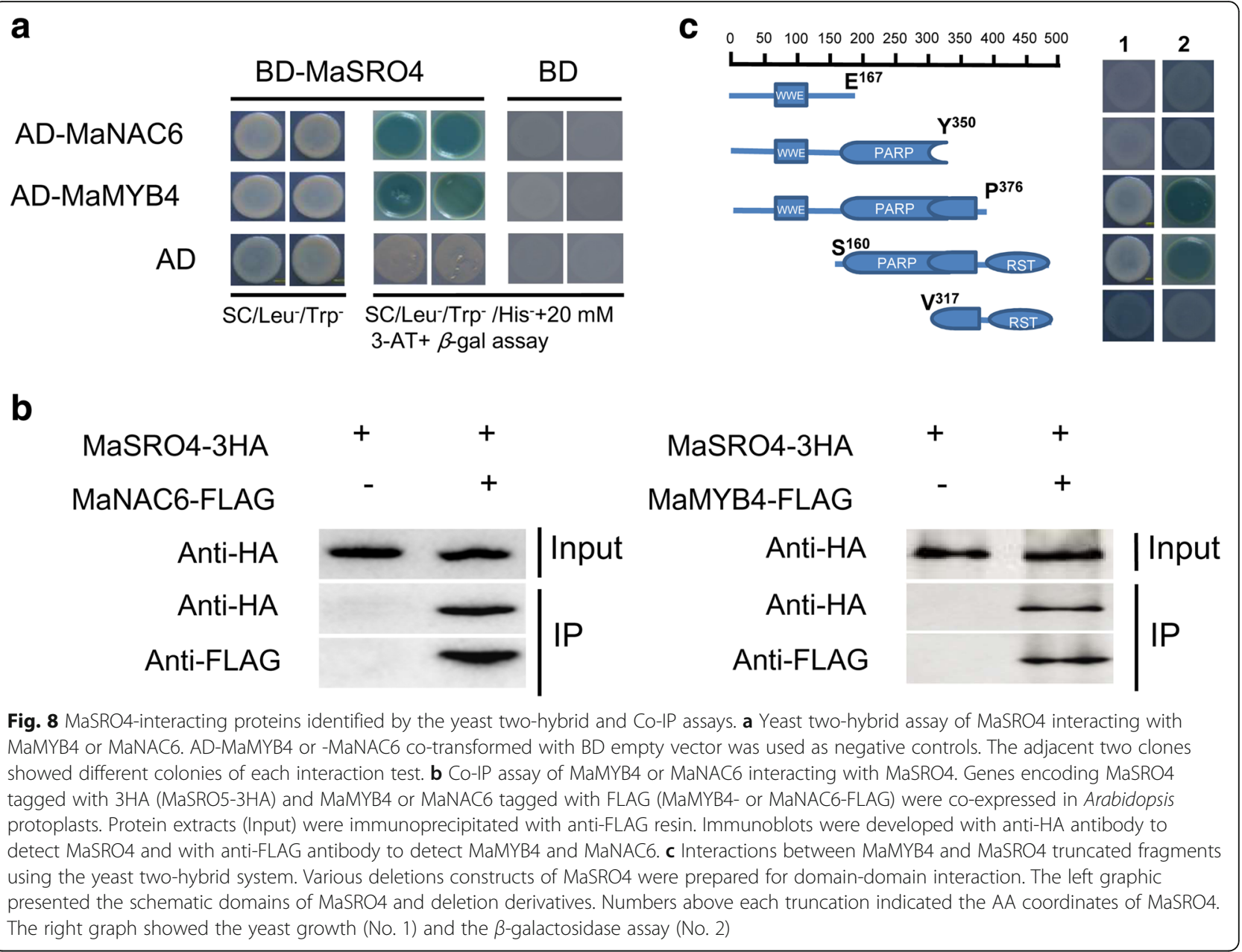

had been characterized to regulate plant development and improve plant's resistance to abiotic stress [13-20]. In our study, six MaSROs were identified in banana genome. The phylogenetic analysis demonstrated that SRO orthologs from the 15 species clustered in the two main groups based on the full-length protein sequences (Fig. 3) and the PARP domain (Additional file 8) [7, 21]. The group II was considered previously as the eudicot group of flowering plants [7], but MaSRO5 and MaSRO6 of banana also clustered into this group. The subgroups (I and II) are distinct with previous classification due to the analysis method and standard [7]. In the subgroup Ia, the selected monocots have at least two SROs except for banana, suggesting that duplication event in the lineage might happen after banana. Actually, the expansion event had happened in $P$. patens. There were three predicted SROs in subgroup Ib. Banana chromosome 4 and 5 contained two MaSRO genes, respectively. It is assumed that maintenance of duplicate genes provides the redundancy function in stress response and plant development, such as AtRCD1 and AtSRO1 [9].
The tissue-specific transcript analysis showed that MaSRO1, 3, 4 and 6 were more abundant in stems and leaves, while MaSRO2 was highly expressed in roots, like their counterparts in rice [20]. The high abundance of $M a S R O$ expression in stems and leaves might participate in the regulation of plant growth and development. Candidates with high transcripts in roots may be important for perceiving various environmental stimuli. We also found that most of MaSROs in the group I were upregulated under PEG treatment (Fig. 4). Likewise, the mutated plants of rice OsSRO1c and Arabidopsis rcd13/sro1-1 demonstrated the increase of stomatal aperture and sensitivity to drought $[15,20]$. It seems that $S R O s$ might be involved in maintenance of cell turgor pressure and decrease of membrane injury under osmotic stress. In addition, qRT-PCR analysis showed that each MaS$R O s$ had at least one response to heat, UV cold, salt, and wounding treatments (Fig. 4). Therefore, different SROs may contribute to diverse abiotic responses. It was supported that the $r c d 1-1$ mutant was more tolerant to UV than the wild-type plants [14]. Compared to the 
function of SROs under abiotic stress, there is little knowledge of SROs in response to biotic stress until now. Previous study indicated that AtRCD1 was involved in the regulation of plant growth, but not in the activation of defense response [29]. We found that more than half of MaSROs displayed differential expression in banana roots inoculated with Foc TR4. Pharmacological inhibition of the PARP protein blocks the plant response to biotic stress induced by microbe associate molecular patterns [30]. In Arabidopsis, loss of PARG1 (poly(ADP-ribose) glycohydrolase) causes greater susceptibility to the necrotrophic pathogen Botrytis cinerea [31, 32]. Hence, MaSROs own different regulation mechanisms under biotic and abiotic stress treatments.

Accumulated evidences indicated that various plant hormones are important for plant's resistance responses to abiotic and biotic stresses $[4-6,13]$. In the present study, most of MaSROs were upregulated by ABA, ethylene and GA treatments. But only MaSRO3 and MaSRO4 exhibited the induced expression under SA treatment (Fig. 5). The previous study showed that abiotic stress responses were largely controlled by $\mathrm{ABA}$, and the priming SA could predominately induce defense against different biotic assailants [3]. It promotes us to speculate that MaSRO3 and MaSRO4 induced by multiple stresses might be involved not only in abiotic stress, but also in biotics stress. Indeed, gene expression depends on the presence of multiple CREs, which integrate signals from diverse TFs to control cell perception and response to environment factors [33]. The ABA response elements (ABREs) were one of the most common CREs in all MaSRO promoters. It is likely consistent with the fact that the transcript accumulations of MaSROs were generally induced by ABA treatment (Fig. 5). Most of the identified CREs within the MaSRO4 promoter could account for its expression levels upregulated by numerous stresses and hormones. Similarly, MaSRO2 are more responsive to ethylene and abiotic stresses, which might be related to the number and type of CREs. By contrast, relatively few CREs were found in the MaSRO1 promoter, corresponding with low or no obvious expression after different treatments (Figs. 4 and 5). Overlapping CREs imply a complexity of gene regulatory network in response to different environmental stimuli. Both ABRE and C-repeat motif/drought-responsive element (CRT/DRE) were also detected in the promoters of cold-responsive genes [34, 35]. The ABRE serves as a coupling factor that functions cooperatively with the CRT/DRE in response to drought and high salinity [33]. Therefore, MaSROs might control a switch in priority between biotic and abiotic stresses by the regulation of a complex hormone network.

Based on transcript characteristics of MaSRO4 induced by multiple stresses and hormone treatments, we analyzed the interaction partners of MaSRO4, which could directly interact with MaMYB4 and MaNAC6 (Fig. 8). Overexpression of a MaMYB4 homolog, OsMYB4, effectively upregulated transcript levels of defense genes and improved stress resistance [36]. NAC was also considered as an interaction center between biotic and abiotic signaling pathways, activated by JA, ethylene, ABA, and SA [37, 38]. Likewise, AtRCD1 or OsSRO1c could interact with a number of TFs in responses to different stress treatments [7, 9]. Interestingly, the AP2 can interact with AtRCD1, AtSRO1 and OsSRO1c [7, 9], but no interaction with their homolog MaSRO4 was detected in the yeast-two-hybrid system. It suggests that functional diversity of the SRO family could be caused by the selective interaction with specific TFs.

To further identify the interaction domain of MaSRO4 with TFs, different $\mathrm{N}$ - and C-terminal truncations were generated. Analysis of conserved AAs supported that the WWE domain could form a half of a beta-barrel with a positively charged PAR-binding pocket [39]. However, the deletion of WWE domain did not affect the interaction of MaSRO5 with MaMYB4 (Fig. 8c), suggesting that the domain is not dispensable for plant's survival. Although the conserved PARP, such as AtRCD1, does not possess ADP-ribosyl transferase activity in plants [7], the PARP domain of MaSRO5 was required for interacting with MaMYB4 (Fig. 8c). The partial or complete loss of the PARP domain within AtRCD1 and AtSRO1 results in a varied expression and a loss of stress tolerance $[9,15]$. It is supported that the functional PARP domain of wheat TaSRO1 could complement the resistance of Arabidopsis plants to salinity stress [21]. However, whether PARP provides allosteric regulation of plant SROs' function still needs to be determined. Compared with the RST domain, the more conservation of amino-acids was found in the C-termini of monocot SROs (Additional file 6). It appears that there are conserved mechanisms of stress resistance in monocots. The RST domain of dicots and monocots likely owns different function in the recruitment of proteins that are involved in activating gene expression. For example, the RST domains of AtRCD1 and AtSRO1 were important for the interaction with TFs $[7,15,16]$. However, the RST domain of MaSRO4 was not necessary for interacting with MaMYB4 in our study (Fig. 8c). Likewise, OsSRO1c without the RST domain still interacts with OsDREB2B [20]. Therefore, these domains of SROs are crucial for the functional diversity and the activation of multiple signaling pathways. How different SROs specifically recognize critical TFs and regulate expression of downstream genes is still unknown. Analysis of motif function, by protein-interaction analyses of TF complexes, is needed. 


\section{Conclusions}

In our present study, six MaSROs were identified and characterized in banana genome. A total of 77 SRO members from 15 species were clustered into two main group with a distinct structure difference. The monocot $S R O s$ were first found in the group II. The qRT-PCR assay showed that half of MaSROs had high expression levels in banana stems and roots. Based on transcript levels induced by diverse stress and hormone treatments, MaSROs might participate in the crosstalk of complex stress signaling pathways. The numbers and types of CREs further support expression profiles of MaSROs. Especially, MaSRO4 with numerous CREs in the promoter region exhibited positive responses to multiple stress treatments, suggesting that it could be a good source for breeding stress-tolerant cultivars. The yeast two-hybrid and Co-IP assays demonstrated that $\mathrm{MaSRO} 4$ could interact directly with MaNAC6 and MaMYB4 through the PARP domain. Therefore, our results provide some novel information to stress-related physiological functions of MaSROs, but their detailed roles still need a number of experimental validation.

\section{Methods}

\section{Plant materials and growth conditions}

Micropropagated banana plantlets of "Williams (Cavendish subgroup, AAA)" and Foc TR4 were obtained from the Chinese Academy of Tropical Agricultural Sciences, Haikou, China. Plantlets were cultured on the Murashige-Skoog medium [40]. Before treatment experiments of biotic and abiotic stresses, micropropagated plants were acclimatized in a secure shade environment as described by Wang et al. (2012) [41].

Identification of SROs and phylogenetic tree construction To identify SROs from 16 different species, sequence files were downloaded from the respective project databases, including banana [42, 43]: the Banana Genome Hub (http://banana-genome-hub.southgreen.fr/, DH Pahang Version 2), Arabidopsis thaliana [44]: the Arabidopsis Information Resource (http://www.arabidopsis.org), Brachypodium distachyon [45]: the Munich Information Center for Protein Sequences (http://mips.helmholtz-muenchen. de/plant/brachypodium, Version 1.2), Medicago truncatula [46]: the HapMap project (http://www.medicagohapmap.org), Oryza sativa [47]: the Rice Genome Annotation Project (http://rice.plantbiol ogy.msu.edu, Version 7.0), Physcomitrella patens [48]: the Physcomitrella patens Resource (http://cosmoss.org/, Version 3.3), Zea mays [49]: the Maize Genetics and Genomics Database (https://www. maizegdb.org/, Version 3.0), and Malus domestica [50]: the Genome Database for Rosaceae (http://www.rosaceae. org/). Two websites (http://www.phytozome.net and https://www.ncbi.nlm.nih.gov/) were used to search the
SROs from Glycine max (Version 2.0) [51], Solanum lycopersicum [52], Setaria italic (Version 2.2) [53], Populus trichocarpa (Version 3.0) [54], Vitis vinifera [55], Phoenix dactylifera [56], Elaeis guineensis [57], and Chlamydomonas reinhardtii [58]. Moreover, the PARP (PF00644) and RST (PF12174) domains from the Pfam database (http:// pfam.xfam.org/, Pfam 32.0 accessed in September 2018) were used as a query to identify all SRO sequences in the respective project databases ( $p$-value $=0.001$ ) [7]. The redundant sequences were removed by the decrease redundancy tool (http://web.expasy.org/decrease_redundancy). The candidate protein sequences were finally confirmed using ScanProsite (https://prosite.expasy.org/ scanprosite/) [59] and SMART (http://smart.embl-heidelberg.de/, accessed on 4 January 2018) [60].

The full-length SRO protein sequences were aligned using a ClustalW program (MEGA version 7.0.1) with default parameters [61]. To compare the domain conservation of SRO evolution, the PARP domain sequences from SROs were also used to do the homology alignment. Two phylogenetic trees were constructed using the Maximum-Likelihood (ML) method of MEGA 7.0.1 with the following parameters: WAG protein substitution model, gamma distribution and bootstrap (1000 replicates). The molecular weight, isoelectric point and grand average of hydrophobicity (GRAVY) of each MaSRO were calculated using the ExPASy website (http://www.expasy.org/tools/) [62].

\section{Prediction of chromosomal location, gene structure and CREs of MaSROs}

The starting and ending positions of all MaSROs on each chromosome were obtained from the banana genome database. The exon-intron structure of each MaSRO was determined by aligning the full-length cDNA sequence with the genomic DNA sequence. The schematic structure of each MaSRO was drawn by the Gene Structure Display Server (http://gsds.cbi.pku.edu. cn/index.php, Version 2.0) [63]. The putative CREs were identified from the $1500 \mathrm{bp}$ upstream sequences of the start codon of each MaSRO using the PlantCARE (http://bioinformatics.psb.ugent.be/webtools/plantcare/ $\mathrm{html} /$ ) [64].

\section{Prediction of conserved motifs and subcellular localizations of MaSROs}

The conserved motifs were detected using a MEME tool (http://meme-suite.org/tools/meme, Version 5.0.4), following the optimum motif width ( $\geqq 10$ and $\leqq 150)$ and seven motifs. The Plant-mPLoc server (http://www.csbio.sjtu.edu.cn /bioinf/plant-multi/, 2.0 version) was used to predict the subcellular localizations of MaSROs [65]. 


\section{Banana seedlings treated with various stresses and inoculated with Foc TR4}

For osmotic and salt stresses, the banana roots of six-leaf seedlings were treated with 15\% of PEG6000 and $200 \mathrm{mM}$ of $\mathrm{NaCl}$ solutions [66], respectively. The samples were collected at $0,3,12$ and $24 \mathrm{~h}$ after osmotic and salt stress treatments. For cold and heat shock stress, seedlings were transferred to a growth chamber at $4{ }^{\circ} \mathrm{C}$ (sampled at $0,3,12$ and $24 \mathrm{~h}$ ) and $42{ }^{\circ} \mathrm{C}$ (sampled at 0 , $0.5,2$ and $4 \mathrm{~h}$ ), respectively. For UV treatment, unfiltered germicidal emitting lamps $(\lambda=254 \mathrm{~nm}$ ) (TUV $15 \mathrm{~W} /$ G15 T8, Phillips, Netherlands) was located $15 \mathrm{~cm}$ above the illumination area for $0,3,6$, and $12 \mathrm{~h}$. Banana root tips were wounded with a scalpel and put on water at room temperature for $0,1,3$ and $6 \mathrm{~h}$. For exogenous hormone treatments, banana roots were cultured in the nutrient solution added with $100 \mu \mathrm{M}$ of ABA, SA, GA3 or ethylene, respectively. The treated roots were sampled after 0, 3, 6 and $12 \mathrm{~h}$. For Foc TR4 inoculation, this strain was cultured on half strength potato dextrose agar at $25^{\circ} \mathrm{C}$ for two weeks. The spores were then centrifuged at $5000 \mathrm{rpm}$ for $5 \mathrm{~min}$ and the pellet was re-suspended in double distilled water. The optical density of fungal suspension was adjusted to $10^{6}$ spores per $\mathrm{ml}$ with sterile water. The roots were dipped into $15 \mathrm{ml}$ of fungal suspension in a Petri dish ( $9 \mathrm{~cm}$ in diameter), and were sampled at 0, 2, 24, 48 and $72 \mathrm{~h}$. All samples were rapidly frozen in liquid nitrogen for RNA isolation. Each experiment was repeated three times.

\section{RNA isolation and qRT-PCR}

Total RNAs of different banana tissues were isolated by RNeasy Plant Mini Kit (Qiagen, Valencia, California, USA). The first strand of cDNAs was synthesized from $2 \mu \mathrm{g}$ of DNaseI-treated total RNA using RevertAid ${ }^{\mathrm{TM}}$ First Strand cDNA Synthesis Kit (Thermo Scientific, USA) according to the manufacturer's instruction. The LightCycler 480 SYBR Green I Master Mix was used for qRT-PCR analysis in a LightCycler 480 System (Roche Diagnositcs, Mannheim, Germany) according to the standard protocol. A total $10 \mu \mathrm{l}$ of reaction solution contained $0.1 \mu \mathrm{l}$ of reverse and forward primers $(100 \mathrm{pmol})$, $1 \mu \mathrm{l}$ of cDNA, $5 \mu \mathrm{l}$ of FastStart SYBR Green I Master Mix and $3.5 \mu$ l of nuclease-free water. The PCR reaction was carried out as follows: $95^{\circ} \mathrm{C}$ for $3 \mathrm{~min}$ and 40 cycles $\left(95^{\circ} \mathrm{C}\right.$ for $10 \mathrm{~s}, 57^{\circ} \mathrm{C}$ for $20 \mathrm{~s}$ and $72{ }^{\circ} \mathrm{C}$ for $20 \mathrm{~s}$ ). The banana $18 S$ rRNA gene (GenBank ID: U42083) was used as an internal control. All reactions were repeated with triple biological replicates. These primer sequences were listed in Additional file 10.

\section{Yeast two-hybrid screening}

The full-length or truncated MaSRO5 was fused to the Gal4 DNA binding domain (BD) in pGBKT7, and was transformed into the yeast strain AH109. The transformants were grown on SD/-Trp plates. The lacZ assay was performed to examine self-activation of the full-length or truncated MaSRO5. The identified yeast strain AH109 harboring the Gal4-BD -MaSRO5 fusion was mated with Y187 (MAT $\alpha)$ containing the Gal4-AD-TF in pGADT7 (GAL4 AD fusion) on 96-well plates. The mating products were plated onto SD/-Trp/ -Leu and SD/-Trp/-Leu/-His/-Ade supplemented with 3-amino-1,4,5-triazole (3-AT). The yeast growth was followed for up to $7 \mathrm{~d}$, and positive colonies were subjected to $\alpha$-galactosidase assay on $\mathrm{SD} /-\operatorname{Trp} /-\mathrm{Leu} /-\mathrm{His} /$ -Ade/X- $\alpha$-Gal. Plates were incubated at $30^{\circ} \mathrm{C}$ and photographed after $5 \mathrm{~d}$ of growth.

\section{Co-immunoprecipitation assay}

Arabidopsis protoplasts were prepared from 4-week-old plants according to the protocol described by Yoo et al. (2007) [67]. MaSRO5-HA was co-expressed with TF-FLAG in Arabidopsis protoplasts. For protein expression, protoplasts $\left(1 \mathrm{ml}, 2 \times 10^{6}\right.$ cells $)$ were transfected with $100 \mu \mathrm{g}$ of plasmid DNA. Transfected protoplasts were incubated in the dark at room temperature for $16 \mathrm{~h}$. The proteins were purified using commercial affinity resins (anti-FLAG M2-resin from Sigma and anti-HA 3F10 resin from Roche). The immuno-precipitates were separated by $10 \%$ SDS-PAGE and proteins on immunoblots were detected with anti-FLAG and anti-HA antibodies [68].

\section{Statistical analyses}

Data processing and statistical analysis were performed with the SPSS statistical software package (SPSS Inc., Cary, NC, USA, v.22). Differences between two treatments were statistically analyzed by the Student's t test.

\section{Additional files}

Additional file 1: The components of amino acids in the conserved motifs. (XLSX $10 \mathrm{~kb}$ )

Additional file 2: Chromosomal location, subcellular localization and putatively physicochemical characters of banana MaSROs. (XLSX $10 \mathrm{~kb}$ )

Additional file 3: Information of the $\mathrm{SRO}$ orthologs in representative sequenced 16 plant species. (XLSX $14 \mathrm{~kb}$ )

Additional file 4: Protein sequences used for the construction of phylogenetic trees. (TXT $39 \mathrm{~kb}$ )

Additional file 5: A newick file of the phylogenetic tree. (TXT $2 \mathrm{~kb}$ ) Additional file 6: Sequence logos of the WWE, PARP, and RST domains in dicots and monocots. The overall height of the stack represented the level of sequence conservation. Heights of residues within a stack indicated the frequency of each residue at the indicated position. (TIF $7444 \mathrm{~kb}$ )

Additional file 7: Distribution of conserved motifs in the SROs. The conserved motifs were identified through the MEME tool. The different colored boxes represented seven motifs. The scale at the bottom was used to estimate the lengths of proteins and motifs location of each motif. (TIF 2906 kb) 
Additional file 8: Unrooted phylogenetic tree of the PARP domain of SROs from 15 plant species. The amino acid sequences of the PARP domain were aligned using Clustal W, and the phylogenetic tree was constructed using MEGA 7.0. (TIF $947 \mathrm{~kb}$ )

Additional file 9: Expression analysis of six MaSROs in banana different tissues by qRT-PCR. Total RNAs were isolated from the roots, stems and leaves of the six-leaf banana seedlings and fruits, respectively. Data indicated relative expression levels (means $\pm \mathrm{SE}$ ) from three independent biological replica (three RNA extractions; $n=3$ ). Used primers of MaSROs were listed in Additional file 10. (TIF $1464 \mathrm{~kb}$ )

Additional file 10: The primer sequences were used in the present study. (XLSX $11 \mathrm{~kb}$ )

\section{Abbreviations}

ABA: Abscisic acid; ABREs: ABA response elements; Co-

IP: Immunoprecipitation; CRT/DRE: C-repeat motif or drought-responsive element; Foc TR4: Fusarium oxysporum f. sp. cubense tropical race 4; hpi: Hour post inoculation; JA: Jasmonic acid; PARP: Poly(ADP-ribose) polymerase: PEG: Polyethylene glycol; qRT-PCR: Quantitative real-time polymerase chain reaction; ROS: Reactive oxygen species; RST: RCD1-SRO-TAF4; SA: Salicylic acid; SRO: SIMILAR TO RCD ONE; TF: Transcription factor; UV: Ultraviolet

\section{Acknowledgements}

Not applicable.

\section{Funding}

Financial support for this project was provided by the National Natural Science Foundation of China (grants 31770476 and 314704651). Publication costs were funded by the China Agriculture Research System (CARS-32). In addition, graduate student salary and tuition support was provided by the Central Public-interest Scientific Institution Basal Research Fund for Chinese Academy of Tropical Agricultural Sciences (1630052019020). The funding bodies had no role in the design of the study and no role in the collection, analysis, and interpretation of data or in writing the manuscript.

\section{Availability of data and materials}

All data generated or analyzed during this study are included in this published article and its supplementary information files.

\section{Authors' contributions}

$L Z, S H, J X$ and WW planned and designed the research; $L Z, D Z, H H, W L, Y H$ performed experiments; LZ, DZ, HH, WL, YH, JX, SH and WW analyzed the data; and LZ, DZ, HH, WL, YH, JX, SH and WW wrote the manuscript. All the authors read and approved the manuscript.

\section{Ethics approval and consent to participate}

Not applicable. Neither human or animal subjects, human or animal materials nor human or animal data were used on this manuscript. The authors declared that experimental research works on the plants described in this paper comply with institutional, national and international guidelines.

\section{Consent for publication}

Not applicable.

\section{Competing interests}

The authors declare that they have no competing interests.

\section{Publisher's Note}

Springer Nature remains neutral with regard to jurisdictional claims in published maps and institutional affiliations.

\section{Author details}

${ }^{1}$ State Key Laboratory of Biocontrol and Guangdong Key Laboratory of Plant Resources, School of Life Sciences, Sun Yat-sen University, Guangzhou 510006, China. ${ }^{2}$ Institute of Tropical Bioscience and Biotechnology, Chinese Academy of Tropical Agricultural Sciences, Haikou 571101, China. ${ }^{3}$ Key Laboratory of Tropical Crop Biotechnology, Ministry of Agriculture, Institute of China Southern Subtropical Crop Research, Chinese Academy of Tropical
Agricultural Sciences, Zhanjiang 524091, China. ${ }^{4}$ School of Life Sciences, Sun Yat-sen University, Guangzhou 510275, Guangdong, China.

Received: 14 February 2019 Accepted: 26 April 2019

Published online: 22 May 2019

\section{References}

1. Lobell DB, Schlenker W, Costa-Roberts J. Climate trends and global crop production since 1980. Science. 2011;333:616-20.

2. Fujita M, Fujita Y, Noutoshi Y, Takahashi F, Narusaka Y, Yamaguchi-Shinozaki $\mathrm{K}$, et al. Crosstalk between abiotic and biotic stress responses: a current view from the points of convergence in the stress signaling networks. Curr Opin Plant Biol. 2006;9:436-42.

3. Apel K, Hirt H. Reactive oxygen species: metabolism, oxidative stress, and signal transduction. Annu Rev Plant Biol. 2004;55:373-99.

4. Mauch-Mani B, Mauch F. The role of abscisic acid in plant-pathogen interactions. Curr Opin Plant Biol. 2005;8:409-14.

5. Lorenzo O, Solano R. Molecular players regulating the jasmonate signalling network. Curr Opin Plant Biol. 2005;8:532-40.

6. Hirayama T, Shinozaki K. Research on plant abiotic stress responses in the post-genome era: past, present and future. Plant J. 2010;61:1041-52.

7. Jaspers P, Overmyer K, Wrzaczek M, Vainonen JP, Blomster T, Salojärvi J, et al. The RST and PARP-like domain containing SRO protein family: analysis of protein structure, function and conservation in land plants. BMC Genomics. 2010;11:170.

8. Belles-Boix E, Babiychuk E, Van Montagu M, Inzé D, Kushnir S. CEO1, a new protein from Arabidopsis thaliana, protects yeast against oxidative damage 1. FEBS Lett. 2000;482:19-24.

9. Jaspers P, Blomster T, Brosche M, Salojärvi J, Ahlfors R, Vainonen JP, et al. Unequally redundant RCD1 and SRO1 mediate stress and developmental responses and interact with transcription factors. Plant J. 2009;60:268-79.

10. Otto H, Reche PA, Bazan F, Dittmar K, Haag F, Koch-Nolte F. In silico characterization of the family of PARP-like poly (ADP-ribosyl) transferases (pARTs). BMC Genomics. 2005;6:139.

11. Hassa PO, Haenni SS, Elser M, Hottiger MO. Nuclear ADP-ribosylation reactions in mammalian cells: where are we today and where are we going? Microbiol Mol Biol Rev. 2006;70:789-829.

12. Aravind $L$. The WWE domain: a common interaction module in protein ubiquitination and ADP ribosylation. Trends Biochem Sci. 2001;26:273-5.

13. Ahlfors R, Lång $S$, Overmyer $K$, Jaspers $P$, Brosché $M$, Tauriainen $A$, et al. Arabidopsis RADICAL-INDUCED CELL DEATH1 belongs to the WWE proteinprotein interaction domain protein family and modulates abscisic acid, ethylene, and methyl jasmonate responses. Plant Cell. 2004;16:1925-37.

14. Fujibe T, Saji H, Arakawa K, Yabe N, Takeuchi Y, Yamamoto KT. A methyl viologen-resistant mutant of Arabidopsis, which is allelic to ozone-sensitive rcd1, is tolerant to supplemental ultraviolet-B irradiation. Plant Physiol. 2004;134:275-85.

15. Teotia S, Lamb RS. The paralogous genes RADICAL-INDUCED CELL DEATH1 and SIMILAR TO RCD ONE1 have partially redundant functions during Arabidopsis development. Plant Physiol. 2009;151:180-98.

16. Teotia S, Lamb RS. RCD1 and SRO1 are necessary to maintain meristematic fate in Arabidopsis thaliana. J Exp Bot. 2010;62:1271-84.

17. Katiyar-Agarwal S, Zhu J, Kim K, Agarwal M, Fu X, Huang A, et al. The plasma membrane $\mathrm{Na}^{+} / \mathrm{H}^{+}$antiporter SOS1 interacts with RCD1 and functions in oxidative stress tolerance in Arabidopsis. Proc Natl Acad Sci U S A. 2006;103:18816-21.

18. Borsani O, Zhu J, Verslues PE, Sunkar R, Zhu JK. Endogenous siRNAs derived from a pair of natural cis-antisense transcripts regulate salt tolerance in Arabidopsis. Cell. 2005;123:1279-91.

19. You J, Zong W, Li X, Ning J, Hu H, Li X, et al. The SNAC1-targeted gene OsSRO1C modulates stomatal closure and oxidative stress tolerance by regulating hydrogen peroxide in rice. J Exp Bot. 2012;64:569-83.

20. You J, Zong W, Du H, Hu H, Xiong L. A special member of the rice SRO family, OSSRO1C, mediates responses to multiple abiotic stresses through interaction with various transcription factors. Plant Mol Biol. 2014;84:693-705.

21. Liu S, Liu S, Wang M, Wei T, Meng C, Wang M, et al. A wheat SIMILAR TO $R C D$-ONE gene enhances seedling growth and abiotic stress resistance by modulating redox homeostasis and maintaining genomic integrity. Plant Cell. 2014;26:164-80. 
22. Li H, Li R, Qu F, Yao J, Hao Y, Wang X, et al. Identification of the SRO gene family in apples (Malus $\times$ domestica) with a functional characterization of MdRCD1. Tree Genet Genom. 2017;13:94.

23. Dale J, James A, Paul JY, Khanna H, Smith M, Peraza-Echeverria S, et al. Transgenic Cavendish bananas with resistance to Fusarium wilt tropical race 4. Nat Commun. 2017:8:1496

24. Pérez Vicente L, Dita M, Martinez De La Parte E. Technical manual prevention and diagnostic of Fusarium wilt (Panama disease) of banana caused by Fusarium oxysporum f. sp. cubense tropical race 4 (TR4). W Diag Fus Wilt. 2014;4:1-74.

25. Van Asten PJA, Fermont AM, Taulya G. Drought is a major yield loss factor for rainfed east African highland banana. Agr Water Manage. 2011;98:541-52.

26. Zhang Q, Zhang JZ, Chow WS, Sun LL, Chen JW, Chen YJ, et al. The influence of low temperature on photosynthesis and antioxidant enzymes in sensitive banana and tolerant plantain (Musa sp.) cultivars. Photosynthetica. 2011;49:201-8.

27. Popper ZA, Michel G, Hervé C, Domozych DS, Willats WG, Tuohy MG, et al. Evolution and diversity of plant cell walls: from algae to flowering plants. Ann Rev Plant Biol. 2011;62(62):567.

28. Kent AG, Dupont CL, Yooseph S, Martiny AC. Global biogeography of Prochlorococcus genome diversity in the surface ocean. ISME J. 2016;10: 1856-65.

29. Zhu Y, Du B, Qian J, Zou B, Hua J. Disease resistance gene-induced growth inhibition is enhanced by $r c d 1$ independent of defense activation in Arabidopsis. Plant Physiol. 2013;161:2005-13.

30. Briggs AG, Adams-Phillips LC, Keppler BD, et al. A transcriptomics approach uncovers novel roles for poly(ADP-ribosyl)ation in the basal defense response in Arabidopsis thaliana. PLoS One. 2017;12:e0190268.

31. Adams-Phillips L, Briggs AG, Bent AF. Disruption of poly(ADP-ribosyl)ation mechanisms alters responses of Arabidopsis to biotic stress. Plant Physiol. 2009;152:267-80.

32. Song J, Keppler BD, Wise RR, Bent AF. PARP2 is the predominant poly(ADPribose) polymerase in Arabidopsis DNA damage and immune responses. PLoS Genet. 2015;11:e1005200

33. Chen WJ, Zhu T. Networks of transcription factors with roles in environmental stress response. Trends Plant Sci. 2004;9:591-6.

34. Zhu T, Provart NJ. Transcriptional responses to low temperature and their regulation in Arabidopsis. Can J Bot. 2003:81:1168-74.

35. Narusaka Y, Nakashima K, Shinwari ZK, Sakuma Y, Furihata T, Abe H, et al. Interaction between two cis-acting elements, abre and dre, in abadependent expression of Arabidopsis rd29a gene in response to dehydration and high-salinity stresses. Plant J. 2010;34:137-48.

36. Vannini C, Iriti M, Bracale M, Locatelli F, Faoro F, Croce P, et al. The ectopic expression of the rice Osmyb4 gene in Arabidopsis increases tolerance to abiotic, environmental and biotic stresses. Physiol Mol Plant Pathol. 2006;69:26-42.

37. Ohnishi T, Sugahara S, Yamada T, Kikuchi K, Yoshiba Y, Hirano HY, et al. OsNAC6, a member of the NAC gene family, is induced by various stresses in rice. Genes Geneti Syst. 2005;80:135-9.

38. Tiwari S, Lata C, Singh Chauhan P, Prasad V, Prasad M. A functional genomic perspective on drought signalling and its crosstalk with phytohormonemediated signalling pathways in plants. Curr Genomic. 2017:18:469-82.

39. Luo X, Kraus WL. On PAR with PARP: cellular stress signaling through poly (ADP-ribose) and PARP-1. Genes Dev. 2012;26:417-32.

40. Murashige T, Skoog F. A revised medium for rapid growth and bioassays with tobacco tissue cultures. Physiol Plantarum. 1962;15:473-97.

41. Wang W, Hu Y, Sun D, Staehelin C, Xin D, Xie J. Identification and evaluation of two diagnostic markers linked to Fusarium wilt resistance (race 4) in banana (Musa spp.). Mol Biol Rep. 2012;39:451-9.

42. Droc G, Lariviere D, Guignon V, Yahiaoui N, This D, Garsmeur O, et al. The banana genome hub. Database. 2013:bat035. https://doi.org/10.1093/database/bat035.

43. Martin G, Baurens FC, Droc G, Rouard M, Cenci A, Kilian A, et al. Improvement of the banana "Musa acuminata" reference sequence using NGS data and semi-automated bioinformatics methods. BMC Genomics. 2016;17(1):243

44. The Arabidopsis Genome Initiative. Analysis of the genome sequence of the flowering plant Arabidopsis thaliana. Nature. 2000;408:796-815.

45. International Brachypodium Initiative. Genome sequencing and analysis of the model grass Brachypodium distachyon. Nature. 2010;463:763-8.

46. Young ND, Debellé F, Oldroyd GE, Geurts R, Cannon SB, Udvardi MK, et al. The Medicago genome provides insight into the evolution of rhizobial symbioses. Nature. 2011:480:520-4.
47. Kawahara Y, de la Bastide M, Hamilton JP, Kanamori H, McCombie WR, Ouyang $S$, et al. Improvement of the Oryza sativa Nipponbare reference genome using next generation sequence and optical map data. Rice. 2013;6:4.

48. Lang D, Ullrich KK, Murat F, Fuchs J, Jenkins J, Haas FB, et al. The Physcomitrella patens chromosome-scale assembly reveals moss genome structure and evolution. Plant J. 2018;93:515-33.

49. Schnable PS, Ware D, Fulton RS, Stein JC, Wei F, Pasternak S, et al. The B73 maize genome: complexity, diversity, and dynamics. Science. 2009:326:1112-5.

50. Jung $\mathrm{S}$, Lee $\mathrm{T}$, Cheng $\mathrm{CH}$, Buble $\mathrm{K}$, Zheng $\mathrm{P}, \mathrm{Yu}$ J, et al. 15 years of GDR: new data and functionality in the genome database for Rosaceae. Nucleic Acids Res. 2018;47:D1137-45.

51. Schmutz J, Cannon SB, Schlueter J, Ma J, Mitros T, Nelson W, et al. Genome sequence of the palaeopolyploid soybean. Nature. 2010;463:178-83.

52. Tomato Genome Consortium. The tomato genome sequence provides insights into fleshy fruit evolution. Nature. 2012;485:635-41.

53. Bennetzen JL, Schmutz J, Wang H, Percifield R, Hawkins J, Pontaroli AC, et al. Reference genome sequence of the model plant Setaria. Nature Biotech. 2012;13(30):555-61.

54. Tuskan GA, Difazio S, Jansson S, Bohlmann J, Grigoriev I, Hellsten U, et al. The genome of black cottonwood, Populus trichocarpa (Torr. \& Gray). Science. 2006;313:1596-604.

55. Jaillon O, Aury JM, Noel B, Policriti A, Clepet C, Casagrande A, et al. The grapevine genome sequence suggests ancestral hexaploidization in major angiosperm phyla. Nature. 2007:449:463-7.

56. Al-Dous EK, George B, Al-Mahmoud ME, Al-Jaber MY, Wang H, Salameh YM, et al. De novo genome sequencing and comparative genomics of date palm (Phoenix dactylifera). Nature Biotech. 2011;29:521-7.

57. Singh R, Ong-Abdullah M, Low ETL, Manaf MAA, Rosli R, Nookiah R, et al. Oil palm genome sequence reveals divergence of interfertile species in old and new worlds. Nature. 2013;500:335-9.

58. Merchant SS, Prochnik SE, Vallon O, Harris EH, Karpowicz SJ, Witman GB, et al. The Chlamydomonas genome reveals the evolution of key animal and plant functions. Science. 2007;318:245-50.

59. Sigrist CJ, De Castro E, Cerutti L, Cuche BA, Hulo N, Bridge A, et al. New and continuing developments at PROSITE. Nucleic Acids Res. 2012;41:D344-7.

60. Letunic I, Bork P. 20 years of the SMART protein domain annotation resource. Nucleic Acids Res. 2017;D1(43):D493-6.

61. Kumar S, Stecher G, Tamura K. MEGA7: molecular evolutionary genetics analysis version 7.0 for bigger datasets. Mol Biol Evol. 2016:33:1870-4.

62. Gasteiger E, Gattiker A, Hoogland C, Ivanyi I, Appel RD, Bairoch A. ExPASy: the proteomics server for in-depth protein knowledge and analysis. Nucleic Acids Res. 2003:31:3784-8.

63. Hu B, Jin J, Guo AY, Zhang H, Luo J, Gao G. GSDS 2.0: an upgraded gene feature visualization server. Bioinformatics. 2014;31:1296-7.

64. Lescot $M$, Déhais $P$, Thijs $G$, Marchal $K$, Moreau $Y$, Van de Peer $Y$, et al. PlantCARE, a database of plant cis-acting regulatory elements and a portal to tools for in silico analysis of promoter sequences. Nucleic Acids Res. 2002;30:325-7.

65. Chou KC, Shen HB. Plant-mPLoc: a top-down strategy to augment the power for predicting plant protein subcellular localization. PLoS One. 2010;5:e11335.

66. Wang Z, Huang S, Jia C, Liu J, Zhang J, Xu B, Jin Z. Molecular cloning and expression of five glutathione S-transferase (GST) genes from Banana (Musa acuminata L. AAA group, cv. Cavendish). Plant Cell Rep. 2013;32:1373-80.

67. Yoo SD, Cho YH, Sheen J. Arabidopsis mesophyll protoplasts: a versatile cell system for transient gene expression analysis. Nat Protoc. 2007;2:1565-72.

68. Cao Y, Liang $Y$, Tanaka $K$, Nguyen $C T$, Jedrzejczak RP, Joachimiak A, Stacey G. The kinase LYK5 is a major chitin receptor in Arabidopsis and forms a chitin-induced complex with related kinase CERK1. Elife. 2014:3:e03766. 